\title{
NREM-sleep Associated Epileptiform Discharges Disappeared Following a Shift toward the Paleolithic Ketogenic Diet in a Child with Extensive Cortical Malformation
}

\author{
Zsófia Clemens $^{1,2, *}$, Anna Kelemen ${ }^{3}$, Csaba Tóth ${ }^{1}$ \\ ${ }^{1}$ Paleomedicina Hungary Ltd., Evolutionary Medicine Working Group, Budapest, Hungary \\ ${ }^{2}$ Neurological Department, University of Pécs, Pécs, Hungary \\ ${ }^{3}$ Department of Neurology, National Institute of Clinical Neuroscience, Budapest, Hungary \\ *Corresponding author: clemenszsofia@gmail.com
}

Received May 30, 2015; Revised June 09, 2015; Accepted June 11, 2015

\begin{abstract}
Purpose: Although the classical ketogenic diet is an effective treatment in childhood epilepsy, it is difficult to maintain on the long term due to side-effects and dislike. Methods: Here we report a case of a child with frequent epileptiform discharges confined to non-rapid-eye-movement (NREM) sleep and extensive cortical malformation. The child was started on a modified version of the classical ketogenic diet we refer to as the paleolithic ketogenic diet. Results: Subsequent follow-up electroencephalograms showed complete normalization of brain electric activity along with cognitive improvement. Neither antiepileptic medication nor vitamin supplements were used. The child strongly adhered to the diet as assessed by regular urinary ketone tests and laboratory work ups. Currently she is on the diet for 17 months. Neither side effects nor clinical signs of nutrient deficiency were observed. Conclusion: We conclude that the paleolithic ketogenic diet was effective, safe and feasible in this case.
\end{abstract}

Keywords: epilepsy, ketogenic diet, paleolithic diet, non-rapid-eye-movement sleep, interictal epileptiform discharges

Cite This Article: Zsófia Clemens, Anna Kelemen, and Csaba Tóth, "NREM-sleep Associated Epileptiform Discharges Disappeared Following a Shift toward the Paleolithic Ketogenic Diet in a Child with Extensive Cortical Malformation.” American Journal of Medical Case Reports, vol. 3, no. 7 (2015): 212-215. doi: 10.12691/ajmcr-3-7-8.

\section{Introduction}

The ketogenic diet has been used in the treatment of epilepsy for 90 years [1]. Several studies, including randomized controlled studies [2], proved its effectiveness which compares to that of antiepileptic drugs while probably confering less side-effects [1]. Current epileptological practice, however, continue to rely on antiepileptic drugs and the ketogenic diet is not recommended as a first-line therapy except for two rare epilepsy syndromes[3]. Previously it was suggested that ketosis represent an evolutionary adapted state in humans [4]. In 1975 gastroenterologist Voegtlin suggested an animal fat-meat based diet as being evolutionarily adapted [5]. Recently we reported a case of a child with absence epilepsy successfully treated with an animal fat-meat based diet we refer to as the paleolithic ketogenic diet [6]. Here we report a case of a child with developmental delay, extensive bilateral cortical malformation and frequent interictal epileptic discharges confined to non-rapid-eyemovement (NREM) sleep electroencephalogram (EEG).The child started the paleolithic ketogenic diet which she strongly adhered to. Follow-up EEGs showed complete normalization of the EEG along with improvements in cognition.

\section{Case Report}

The child was born at full term with no complications in 2007. A routine ultrasound examination at 10 days old showed enlarged ventricles of the brain. Her muscles were spastic and she had difficulties with feeding. A repeated ultrasund examination at 1 year old showed moderately enlarged ventricles but no signs of intracranial hypertension. An MRI examination performed in October 2012 showed extensive bilateral polymicrogyria involving all brain lobes except for the frontal lobe. The MRI also indicated septal agenesia and hypogenesis of the corpus callosum. Additional dismorphic features included microcephaly, hypertelorism, arched palate and dysmorphic auricles. Genetic tests for subtelomeric deletion, Angelman syndrome and congenital glycosylation disorder were negative. Due to a delay in motor development she has been receiving movement therapy. She had a complex cognitive developmental 
delay including attention deficit an inability to speak. She has also been receiving interventions to improve cognition. A whole-night EEG performed on 02 Aug 2013 showed frequent multifocal epileptiform discharges confined to non-rapid-eye-movement (NREM) sleep (Figure 1). Interictal epileptic discharges were continuous during NREM stage 2 and were very frequent during NREM stage 3 and 4 . No epileptiform discharges were seen during wakefulness. The child had no history of ouvert seizures. Parents of the child refused antiepileptic medication and decided to initiate the paleolithic ketogenic diet. The child shifted from the normal diet toward the paleolithic ketogenic diet gradually within one month. In December 2013 multiple fasciotomy was performed at a foreign clinic to alleviate muscle contractures. From 01 January 2014 the child was on a full paleolithic ketogenic diet. The diet mostly consisted of animal fat, meat, offal with a fat:protein ratio of at least $2: 1$. Fat and red meats as well as offal from pork and cattle were encouraged. She was allowed to consume vegetables to an extent which did not prevent ketosis as regularly assessed by urinary ketone test. Although we did not recommend it, she also used coconut flour in small amounts to make the diet more palatable. No plant oil of any form were allowed. Artificial sweeteners were restricted but small amounts of honey was allowed. At diet initiation we advised against taking vitamin and mineral supplements and so the child stopped all supplements she was taking before. Ketosis was regularly checked by urinary ketone strips which indicated sustained ketosis. Laboratory check-ups were carried out five times during the 17 months on the diet. All five urinary laboratory tests were positive for ketones. Avarage blood glucose was $3.98( \pm 0.42) \mathrm{mmol} / \mathrm{l}$. Blood count, renal and liver function were normal. Inflammatory markers, including CRP and fibrinogen, as well as triglicerides and uric acid remained low. Total cholesterol and LDL were elevated (Table 1). On 02 February 2014, four weeks after diet onset, a whole-night sleep EEG was performed. This showed normal sleep structure and the absence of clear-cut epileptiform activity. The EEG only showed infrequent waveforms during NREM sleep that were sharper in morphology (Figure 2). As before no EEG abnormalities were seen during wakefulness. Four months later on 14 Jun 2014 a next whole-night EEG recording showed normal sleep structure, normal background activity and the absence of epileptiform activity (Figure 3).

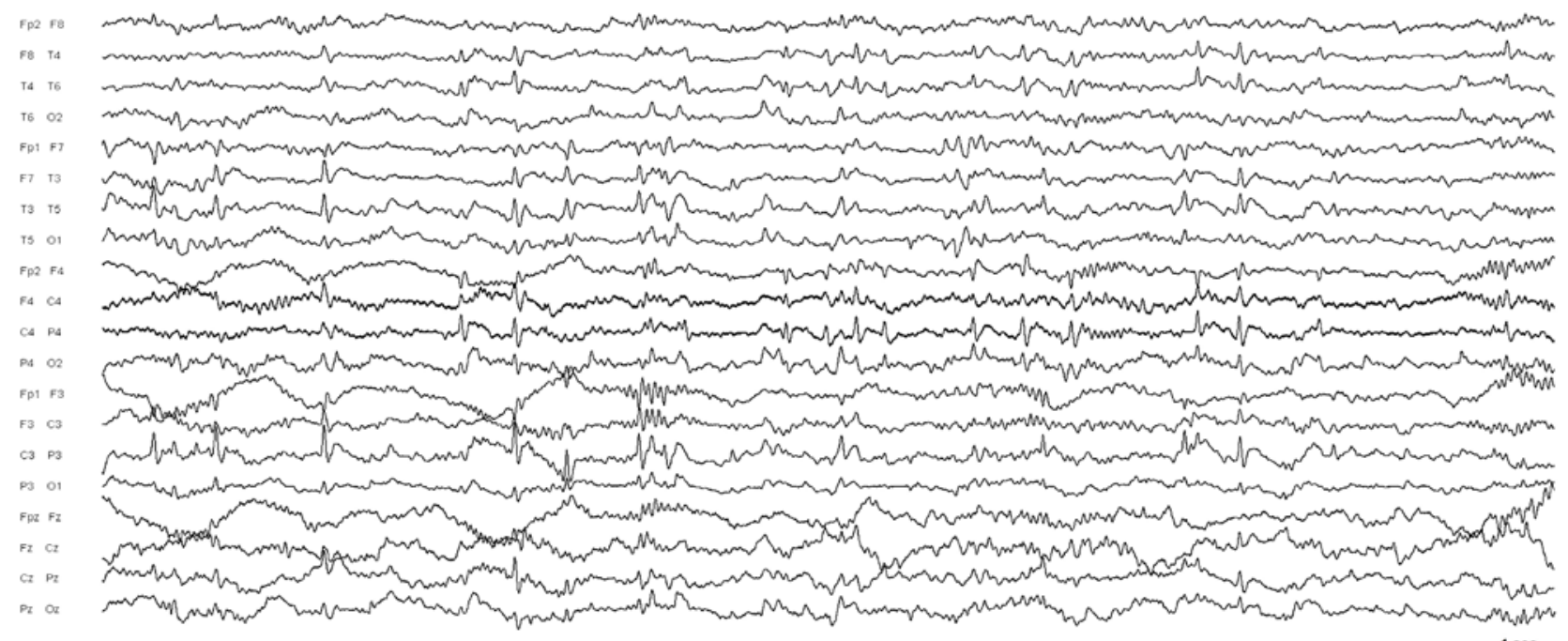

$1 \mathrm{sec}$

Figure 1. EEG during NREM stage 2 sleep recorded on 02 Aug 2013 while on a normal diet. Note frequent multifocal epileptiform activity

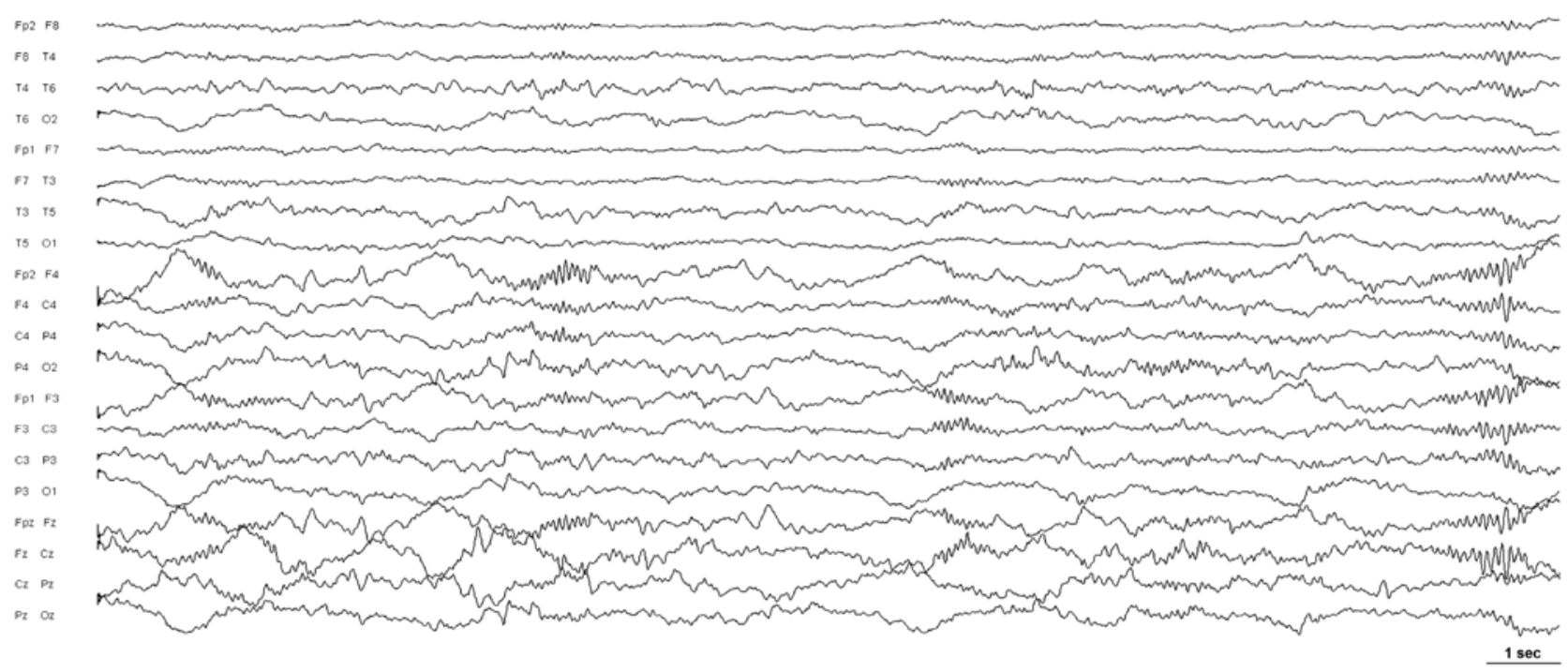

Figure 2. EEG during NREM stage 2 sleep recorded on 02 Feb 2014 four weeks after the onset of the paleolithic ketogenic diet. Normal EEG 


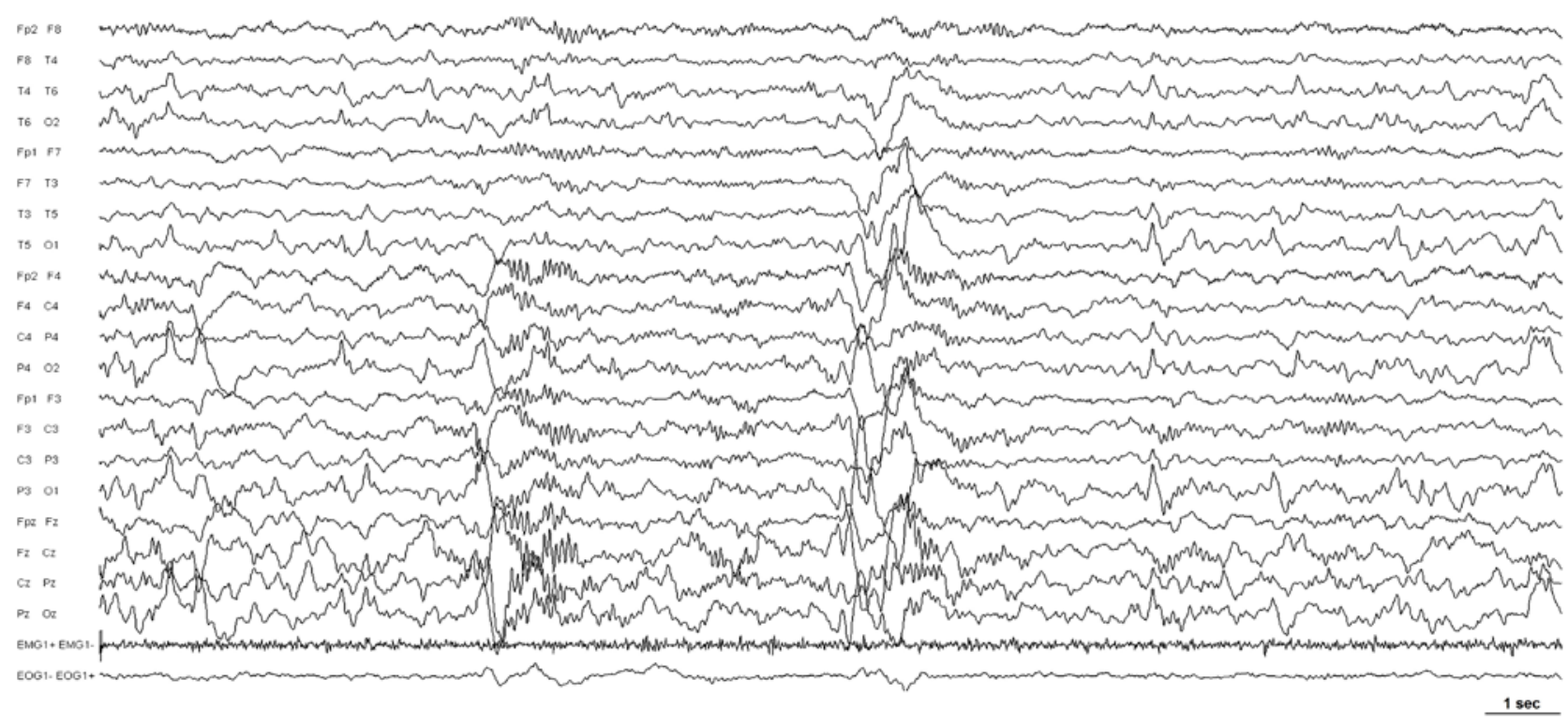

Figure 3. EEG during NREM stage 2 sleep recorded on 14 Jun 2014 six months after the onset of the paleolithic ketogenic diet. Normal EEG

Table 1. Laboratory data while on a normal diet (on 11 November 2013) and on the paleolithic ketogenic diet 13 months later (on 13 December 2014). Note that except for cholesterol and LDL cholesterol all parameters fall in the normal range while on the paleolithic ketogenic diet

\begin{tabular}{|c|c|c|c|}
\hline Normal diet & \multicolumn{3}{|c|}{ Paleolithic-ketogenic diet } \\
\hline Sodium & 140 & 138 & $\mathrm{mmol} / \mathrm{l}$ \\
\hline Potassium & 4.5 & 4.7 & $\mathrm{mmol} / \mathrm{l}$ \\
\hline Calcium & 2.58 & 2.42 & $\mathrm{mmol} / \mathrm{l}$ \\
\hline Magnesium & 0.83 & 0.9 & $\mathrm{mmol} / \mathrm{l}$ \\
\hline Carbamide & 6.6 & 3.4 & $\mathrm{mmol} / \mathrm{l}$ \\
\hline Creatinine & 33 & 23 & $\mu \mathrm{mol} / 1$ \\
\hline Glucose & 3.9 & 3.9 & $\mathrm{mmol} / \mathrm{l}$ \\
\hline HgA1c & 5.2 & 4.8 & $\%$ \\
\hline Total cholesterol & 6.1 & 12.3 & $\mathrm{mmol} / \mathrm{l}$ \\
\hline HDL cholesterol & 1.57 & 1.44 & $\mathrm{mmol} / \mathrm{l}$ \\
\hline LDL cholesterol & 4.23 & 10.85 & $\mathrm{mmol} / \mathrm{l}$ \\
\hline Triglyceride & 0.49 & 0.76 & $\mathrm{mmol} / \mathrm{l}$ \\
\hline Uric acid & 168 & 209 & $\mu \mathrm{mol} / 1$ \\
\hline Total protein & 77 & 73 & $\mathrm{G} / \mathrm{l}$ \\
\hline WBC & 5.4 & 4.9 & $\mathrm{G} / \mathrm{l}$ \\
\hline $\mathrm{RBC}$ & 4.8 & 4.4 & $\mathrm{~T} / \mathrm{l}$ \\
\hline Iron & 6.1 & 17.8 & $\mu \mathrm{mol} / 1$ \\
\hline Hemoglobin & 124 & 126 & $\mathrm{~g} / \mathrm{l}$ \\
\hline GOT & 30 & 28 & $\mathrm{U} / \mathrm{l}$ \\
\hline GPT & 13 & 16 & $\mathrm{U} / \mathrm{l}$ \\
\hline GGT & 11 & 13 & $\mathrm{U} / \mathrm{l}$ \\
\hline ALP & 220 & 197 & $\mathrm{U} / \mathrm{l}$ \\
\hline fibrinogen & 3.59 & 2.44 & $\mathrm{~g} / \mathrm{l}$ \\
\hline CRP & 0.3 & 0.1 & $\mathrm{mg} / \mathrm{l}$ \\
\hline
\end{tabular}

Abbreviations: HgA1c - glycated hemoglobin, HDL - high density lipoprotein, LDL - low density lipoprotein, WBC - white blood cell count, RBC - red blood cell count, GOT - glutamate-oxaloacetate transaminase, GPT - glutamate-pyruvate transaminase, GGT - gammaglutamyl transferase, ALP - alkaline phosphatase, CRP - C-reactive protein

As reported by the parents adhering to the diet was relatively easy for the child. Currently she is on the paleolithic ketogenic diet for 17 months. The parents of the child are committed to continue the diet. Neither gastrointestinal nor other side effects emerged. Also, no clinical signs of vitamin or mineral deficiencies emerged.

Parents of the child gave written informed consent for publication of this case.

\section{Discussion}

Sleep, and especially NREM sleep, is critically involved in a variety of cognitive functions $[7,8]$. Frequent epileptic activity during NREM sleep is thought to deteriorate sleep-related cognitive processing in epileptic children [9]. Landau-Kleffner syndrome is a form of epilepsy presenting without seizures but with epileptic activity over the sylvian region resulting is a loss of language skills [10]. Our patient, however, may not be classified as having Landau-Kleffner syndrome given the more widespread morphological and EEG abnormalities and the complexity of her developmental delay.

The parents of the child refused antiepileptic medication and therefore the paleolithic ketogenic diet was initiated as a stand-alone therapy. Follow up sleepEEG performed at four weeks on the diet showed the absence of clear-cut epileptiform discharges during NREM sleep. A next sleep-EEG performed at five months after diet onset showed no epileptiform EEG activity at all. Parents and caretakers of the child noted improvements in cognition including learning, concentration, speech comprehension as well as improvements in vocalization and motor skills. Parent reports, home monitoring of urinary ketone as well as laboratory check-ups showed an excellent adherence to the diet and sustained ketosis. Her laboratory parameters were normal except for total cholesterol and LDL cholesterol which were elevated. In our previous case with absence epilepsy [6] and in another case of type 1 diabetes [11] on the paleolithic ketogenic diet these two laboratory measures were also elevated although not to this extent. Elevation of cholesterol is also known from studies with the classical ketogenic diet [12]. Yet it is now acknowledged that neither serum nor dietary cholesterol should be regarded as a risk factor for cardiovascular disease [13]. Instead, other laboratory 
parameters including C-reactive protein [14] and fibrinogen [15] have been suggested as better predictors of cardiovascular disease. Of note, these latter measures were consistently low in our patient. We speculate that in our patient elevated cholesterol may reflect ongoing regenerative processes. Other laboratory parameters including uric acid and triglycerides were normal like in our other patients on the paleolithic ketogenic diet $[6,11,16]$. Adverse effects known to be associated with the classical ketogenic diet such as iron-deficiency anemia, hypomagnesemia, increased frequency of infections and gastrointestinal side effects [17] did not emerge in our patient on the paleolithic ketogenic diet despite of the absence of supplementation. Of note, no such adverse effects were seen in our previous cases on the paleolithic ketogenic diet $[6,11,16]$. It is worth noting that the complete normalization of the EEG, in terms of disappearence of interictal epileptiform spikes, seen in our both epilepsy cases on the paleolithic ketogenic diet is very rare among patients on the classical ketogenic diet [18].

At the time of writing this manuscript the patient is on the diet for 17 months. We believe that the paleolithic ketogenic diet may be remedial while confering neither side effects nor deficiencies because it is of full nutritional value matching the human evolutionary needs $[19,20]$.

\section{Compliance with Ethical Standards}

The authors declared no potential conflicts of interest. The case study was done in accordance with the Helsinki Declaration of 1975. Informed consent was obtained the parents of the child. The authors received no financial support for the research.

\section{References}

[1] Kossoff $\mathrm{EH}(2004)$ More fat and fewer seizures: dietary therapies for epilepsy. Lancet Neurol 3:415-20.

[2] Levy RG, Cooper PN, Giri P (2012) Ketogenic diet and other dietary treatments for epilepsy. Cochrane Database Syst Rev3:CD001903.
[3] Kossoff EH (2008) International consensus statement on clinical implementation of the ketogenic diet: agreement, flexibility, and controversy. Epilepsia 49 Suppl 8:11-3.

[4] Fine EJ, Segal-Isaacson CJ, Feinman R, Sparano J (2008) Carbohydrate restriction in patients with advanced cancer: a protocol to assess safety and feasibility with an accompanying hypothesis. Commun Oncol 5:22-6.

[5] Voegtlin WL (1975) The stone age diet: based on in-depth studies of human ecology and the diet of man. Vantage Press,New York

[6] Clemens Z, Kelemen A, Fogarasi A, Tóth C (2013) Childhood Absence Epilepsy Successfully Treated with the Paleolithic Ketogenic Diet. Neurology and Therapy 2:71-6.

[7] Gais S, Born J (2004) Declarative memory consolidation: mechanisms acting during human sleep. Learn Mem11:679-85.

[8] Clemens Z, Fabó D, Halász P (2005) Overnight verbal memory retention correlates with the number of sleep spindles. Neuroscience 132:529-35.

[9] Holmes GL, Lenck-Santini PP (2006) Role of interictal epileptiform abnormalities in cognitive impairment. Epilepsy Behav 8:504-15.

[10] Halász P (2013) How Sleep Activates Epileptic Networks? Epilepsy Res Treat 2013:425697.

[11] Tóth C, Clemens Z (2014) Type 1 diabetes mellitus successfully managed with the paleolithic ketogenic diet. Int J Case Rep Images5:699-703.

[12] Liu YM, Lowe H, Zak MM, Kobayashi J, Chan VW, Donner EJ (2013) Can children with hyperlipidemia receive ketogenic diet for medication-resistant epilepsy? J Child Neurol 28:479-83.

[13] Lecerf JM, de Lorgeril M (2011) Dietary cholesterol: From physiology to cardiovascular risk. Br J Nutr 106:6-14.

[14] Lagrand WK, Visser CA, Hermens WT, Niessen HW, Verheugt FW, Wolbink GJ, Hack CE (1999)C-reactive protein as a cardiovascular risk factor: more than an epiphenomenon? Circulation. 100:96-102.

[15] Stec JJ, Silbershatz H, Tofler GH, Matheney TH, Sutherland P, Lipinska I, Massaro JM, Wilson PF, Muller JE, D'Agostino RB Sr (2000) Association of fibrinogen with cardiovascular risk factors and cardiovascular disease in the Framingham Offspring Population. Circulation102:1634-8.

[16] Tóth C, Clemens Z (2015) Successful treatment of a patient with obesity, type 2 diabetes and hypertension with the paleolithic ketogenic diet. International Journal of Case Reports and Images (accepted for publication).

[17] Kang HC, Chung DE, Kim DW, Kim HD (2004) Early- and lateonset complications of the ketogenic diet for intractable epilepsy. Epilepsia 45: 1116-23.

[18] Remahl S, Dahlin MG, Amark PE (2008) Influence of the ketogenic diet on 24-hour electroencephalogram in children with epilepsy. Pediatr Neurol. 38:38-43.

[19] Eaton SB, Konner M (1985) Paleolithic nutrition. A consideration of its nature and current implications. N Engl J Med312:283-9.

[20] Cordain L (2002) The paleo diet: lose weight and get healthy by eating the food you were designed to eat. WileyNew, York. 